\title{
Effect of Hydrogen Bonding on Self-diffusion in Methanol/Water Liquid Mixtures: A Molecular Dynamics Simulation Study
}

\author{
Ning Zhang ${ }^{\mathrm{a}}$, Zhuanglin Shen ${ }^{\mathrm{a}}$, Cong Chen ${ }^{\mathrm{b}}$, Gaohong He${ }^{\mathrm{a}}{ }^{*}, \mathrm{Ce} \mathrm{Hao}^{\mathrm{a}}$ \\ ${ }^{a}$ State Key Laboratory of Fine Chemicals, School of Petroleum and Chemical Engineering, \\ Dalian University of Technology, Panjin 124221, China \\ ${ }^{\mathrm{b}}$ Key Laboratory of Ocean Energy Utilization and Energy Conservation of Ministry of Education, \\ Dalian University of Technology, Dalian 116024, China \\ * Corresponding author. Tel.: +86-427-2631518. \\ E-mail address: zhangning@dlut.edu.cn
}

\begin{abstract}
Self-diffusion motion is strongly dependent on hydrogen bonding and temperature. In this work, the methanol/water mixtures of molalities from 1 to $5 \mathrm{~m}$ have been studied by molecular dynamics simulation. A definition of hydrogen bonding state is proposed to detailedly analyze the effects of the type and number of hydrogen bonds on the water self-diffusion. It is shown that most water molecules are in the hydrogen bonding state $f_{k}^{k}$, which means that one water molecule simultaneously hydrogen bonded to $k$ water molecules with one hydrogen bond, respectively. Methanol prefers to produce isolated water molecules by the insertion of its hydrophobic group into the hydration shell of water. Besides, calculation of mean square displacements of water in different hydrogen bonding states shows that one water molecule with more hydrogen bonds diffuses more slowly. Pair energy of the hydrogen bonded molecules are also calculated to compare the attractive interactions of different types of hydrogen bonds. The hydrogen bonds between methanol and water present stronger attraction than that between water molecules. It indicates that
\end{abstract}


increasing the concentration of methanol is conductive to restricting the water self-diffusion. Cluster analysis reveals that methanol cluster is more stable than water cluster in the binary mixtures. Thus high concentration of methanol enhances the blockage of the methanol cluster to water movement. These findings will lead us to further understand the mechanisms of water self-diffusion in the methanol/water mixture.

Keywords: Molecular dynamics simulation, Hydrogen bonding state, Self-diffusion, Methanol/water mixture

\section{Introduction}

In aqueous solutions, amphiphiles exhibit attractive and powerful ability of organizing the local structure where the amphiphilic molecules tend to be pushed together by the low affinity of water to the hydrophobic groups, and hydrogen bond (H-bond) to the surrounding water molecules by hydrophilic groups[1]. The H-bonds between amphiphilic molecules and water is a substitution of the $\mathrm{H}$-bonds between water molecules, resulting in the rearrangement of the local hydrogen bonding (H-bonding) structure. Methanol is one of the simplest amphiphiles with both hydrophobic and hydrophilic groups. It is widely used in the fields of organic synthesis, biochemistry and biotechnology. Moreover, the development of direct methanol fuel cell leads to a growing interest in the studies of the microscopic properties of methanol[2-4]. Therefore, it is important to acquire a deep comprehension of the methanol/water mixture properties. The hydroxyl group of each methanol molecule can simultaneously act as H-bond donor and acceptor, and the methyl group has a negative effect on the formation of H-bonds[5]. The structure arrangement of the methanol/water mixtures has close correlation with the local hydration structure and dynamics of the amphiphilic solutes in the polar solvent $[1,6]$. Thus the tetrahedral-like H-bonding network formed by water molecules is apt to be interrupted by the presence of methanol[7]. Although methanol is soluble in water, their binary mixtures are incompletely mixed at the microscopic scale resulted from 
the formations of water and methanol clusters[6]. Some interesting non-ideal properties of methanol/water mixtures are considered to be correlated to intermolecular interaction such as H-bonding[8].

Investigations of the effect of methanol on the structure and dynamics of its aqueous solutions have been carried out in various fields by experiments and simulations. Dougan et al.[9] employed both neutron diffraction experiment and molecular dynamics simulation to investigate the structural properties of methanol/water mixtures with various proportions. The results show that the H-bonding and hydrophobic interactions lead to a bi-percolating network near the molar fraction of 0.27 , where many properties such as transport coefficients and thermodynamic functions present abnormal values. The bi-percolating network in methanol/water mixtures was also found by Zhong and coworkers[8].

$\mathrm{H}$-bonds play a determining role in understanding the microscopic structural and dynamical properties of the aqueous solutions, thus numerous studies[10-13] provide the information of the H-bonding effect on several properties including self-diffusivity. Molecular dynamics (MD) simulation has become a widely used tool for calculating self-diffusion coefficients[14, 15], and efforts have been devoted to the application of molecular-level techniques to macroscopic evolution equations[16, 17]. The mechanisms of the dependences of water self-diffusion motion on concentration and temperature are still open to question. In our previous study[6], the diffusion motion of methanol and water molecules was found to be germane to the intermolecular interaction of H-bonding in their binary mixtures. The mean square displacements of water molecules in different $\mathrm{H}$-bonding states were calculated to quantitatively evaluate the H-bonding effect on the self-diffusion motion. The results revealed that one water molecule with high content of H-bonds has low self-diffusion coefficient, resulting in high viscosity in the macroscopic view. However, different types of H-bonds were not specifically considered in the statistical and dynamical analysis for each H-bonding state. There are two probable types of H-bonds (i.e. water-water (W-W) and methanol-water (W-M) H-bonds) presenting in the methanol/water mixtures. For example, one water molecule in the H-bonding state $f_{1}$ denotes that one 
water molecule initially with one H-bond, where $f_{1}$ is the collection of one water molecule hydrogen bonded (H-bonded) to another water or methanol molecule; and three probable combinations (i.e. $2 \mathrm{~W}-\mathrm{W}, 2 \mathrm{~W}-\mathrm{M}$ and $1 \mathrm{~W}-\mathrm{W}+1 \mathrm{~W}-\mathrm{M}$ H-bonds) may exist in the H-bonding state $f_{2}$, etc[6]. The previous paper[6] only presented a mean effect of different types of H-bonds on the water self-diffusion, thus each H-bonding state should be subdivided to analyze the effects of different H-bonding types.

In this work, we will propose a definition, by which the contribution of two types of H-bonds (i.e. W-W and W-M H-bonds) to the water molecules of different H-bonding states could be distinguished. By means of the definition, the effects of temperature and concentration on the water molecules of different $\mathrm{H}$-bonding states are investigated by calculating the distributions of the corresponding H-bonding states. The paper also studies the self-diffusion motion of the water molecules in different H-bonding states. An in-depth analysis is made to elucidate the relation between self-diffusion and H-bonding state, and explore which hydrogen bonding state is the dominant role in retarding the water self-diffusion. Then the pair energies of the $\mathrm{W}-\mathrm{W}$ and $\mathrm{W}-\mathrm{M} \mathrm{H}$-bonded pairs are calculated to interpret the effect of $\mathrm{H}$-bonding interaction on the water self-diffusion.

\section{Computational details}

Table.1 Compositions of the water and methanol molecules in different systems

\begin{tabular}{cccc}
\hline$m(\mathrm{~m})$ & $x(\%)$ & $N_{\mathrm{m}}$ & $N_{\mathrm{w}}$ \\
\hline 1 & 1.8 & 50 & 2803 \\
2 & 3.4 & 75 & 2102 \\
3 & 5.1 & 80 & 1496 \\
4 & 6.7 & 90 & 1150 \\
5 & 8.2 & 100 & 1121 \\
\hline
\end{tabular}

$m$ denotes molality of methanol in aqueous solution; $x$ is the percentage of mass fraction of methanol; $N_{\mathrm{m}}$ and $N_{\mathrm{w}}$ are the numbers of methanol and water molecules, respectively.

Classical MD simulations using the package NAMD 2.7 were performed in the 
isothermal-isobaric (NpT) ensemble for the methanol/water mixtures of different compositions. The compositions of the mixtures are denoted by molality ranging from 1 to $5 \mathrm{~m}$, which are summarized in Table 1 . The methanol/water mixtures were simulated under the conditions of the pressure $1 \mathrm{~atm}$ and the three different temperatures (273, 288 and $298 \mathrm{~K})$. TIP3P potential[18] was used as the model of water. Force fields described by Mackerell et al.[19] was adopted to model methanol. Table 2 provides the results of the densities of the simulation systems at $288 \mathrm{~K}$.

Table. 2 Comparison between the simulation and experimental results of the density for different systems at $288 \mathrm{~K}$

\begin{tabular}{cccc}
\hline$m(\mathrm{~m})$ & $\rho_{\text {sim }}(\mathrm{g} / \mathrm{ml})$ & $\rho_{\exp }(\mathrm{g} / \mathrm{ml})$ & $\left(\rho_{\text {sim }}-\rho_{\exp }\right) / \rho_{\exp }(\times 100 \%)$ \\
\hline 1 & 1.0101 & 0.9958 & 1.4 \\
2 & 1.0013 & 0.9930 & 0.8 \\
3 & 0.9940 & 0.9901 & 0.4 \\
4 & 0.9170 & 0.9875 & -7.1 \\
5 & 0.9809 & 0.9852 & -0.4 \\
\hline
\end{tabular}

The experimental data for density of the methanol/water mixtures are given by the reference.

All simulations were performed under periodic boundary conditions in three dimensions to minimize the edge effect. The temperatures and pressure were controlled using Langevin dynamics and Langevin piston Nosé-Hoover methods to fluctuate around the abovementioned values, respectively. The particle mesh Ewald (PME) method was used to solve the full electrostatic interactions, in which the grid spacing was set to $1.0 \AA$. The cut-off distances of the switching and shifting functions were both set to $12 \AA$, which specified the distances where van der Waals interaction was truncated and the long and short range forces were separated for electrostatic interactions. The SHAKE algorithm was employed to constrain the covalent bond between hydrogen atom and its bonded atom around the nominal length.

For each simulation, the methanol/water system was first optimized with energy minimization, and the time step was set to $2.0 \mathrm{fs}$. The equilibration phase of each system was continued to run until the total energy of the system appeared to plateau. 
Equilibration took about $2.0 \mathrm{~ns}$ to reach. After equilibration of the system was reached, a production run was carried out for $3.0 \mathrm{~ns}$ to analyze the microscopic properties, during which the sampling frequency was set to once per 1000 time steps. For the calculation of mean square displacement (MSD), a subsequent production run for 200 ps was performed with sampling frequency of once per 10 time steps.

\section{Results and discussion}

\subsection{Statistical analysis of H-bonding}

\subsubsection{Definition of H-bonds}

Definition of $\mathrm{H}$-bonds should be determined prior to the $\mathrm{H}$-bonding analysis of the methanol/water systems. Following the previous studies[6, 20], two H-bonded molecules should fulfill the geometric criterion involving the three conditions below:

(1) The distance $R_{\mathrm{OO}}$ between donor and acceptor should shorter than the threshold value $R_{O O}^{C}$;

(2) The distance $R_{\mathrm{OH}}$ between acceptor and donor hydrogen should shorter than the threshold value $R_{O H}^{C}$;

(3) The angle $\mathrm{O}-\mathrm{H} \cdots \mathrm{O}$ should smaller than the threshold value $\varphi^{C}$.

The threshold values $R_{O O}^{C}$ and $R_{O H}^{C}$ are determined from the positions of the first minima of the corresponding radial distribution functions (RDFs). The threshold values $R_{O O}^{C}$ and $R_{O H}^{C}$ are determined as at 3.5 and $2.45 \AA$ [21] (not presented for brevity). The threshold value $\varphi^{C}$ is defined as the widely accepted value 30 [1, 6]. We also checked the variations of the threshold value $\varphi^{C}$ up to $70^{\circ}$, and observed negligible changes of the average number of $\mathrm{H}$-bonds and their probability distribution with $\varphi^{C}$ larger than $30^{\circ}$. 


\subsubsection{Definition of H-bonding state}

In the methanol/water mixtures, there are six H-bonding states for water molecules as reported in the previous study[6]. Except for the H-bonding state $f_{0}$, each H-bonding state could be composed of different combinations. Herein, a quantity $f_{k}^{n}$ is defined to detailedly describe the constituents of each $\mathrm{H}$-bonding state of water, where $k$ denotes the total number of $\mathrm{H}$-bonds one water molecule has, and $n$ denotes the number of water molecules H-bonded to the same water. In other words, one water molecule with the H-bonding state $f_{k}^{n}$ means that it has $n \mathrm{~W}-\mathrm{W} \mathrm{H}$-bonds and $(k-n)$ $\mathrm{W}-\mathrm{M} \mathrm{H}$-bonds. It is found that the maximum number of the $\mathrm{H}$-bonds one water molecule can form is 5 , thus the upper limit of the value $k$ is set to 5 .

\subsubsection{Distribution of different $\mathrm{H}$-bonding states}

On the basis of the abovementioned H-bonding scheme, the constituents of each H-bonding state are calculated with the previously reported geometric criterion of H-bonds[6]. Table 3 gives the results of $f_{k}^{n}$ in the methanol/water mixture with the concentration of $5 \mathrm{~m}$ at $298 \mathrm{~K}$. It should be noted that water molecules are interested to form three H-bonds, among which the type of $\mathrm{W}-\mathrm{W}$ H-bonds (i.e. $f_{3}^{3}$ ) takes the dominant role. It is revealed that the binary mixtures retain the local tetrahedral structure in the studied concentration range. This phenomenon can be also observed in other methanol/water mixtures of the concentrations from 1 to $4 \mathrm{~m}$. For the sake of brevity, the percentages of the water molecules in different H-bonding states for the mixtures with the concentrations $1 \sim 4 \mathrm{~m}$ at $298 \mathrm{~K}$ are shown in the supplemental information (Table S1).

Table 3. Percentages $\left(P\left(f_{k}^{n}\right) \%\right)$ of the water molecules at different $\mathrm{H}$-bonding states

$\left(f_{k}^{n}\right)$ in the methanol/water mixture with $5 \mathrm{~m}$ at $298 \mathrm{~K}$

$\widehat{k}^{n}$




\begin{tabular}{ccccccc}
\hline 0 & 1.494 & - & - & - & - & - \\
1 & 0.583 & 9.532 & - & - & - & - \\
2 & 0.092 & 2.867 & 22.833 & - & - & - \\
3 & 0.008 & 0.405 & 5.813 & 29.498 & - & - \\
4 & 0.000 & 0.018 & 0.496 & 5.009 & 18.848 & - \\
5 & 0.000 & 0.000 & 0.004 & 0.062 & 0.571 & 1.867 \\
\hline
\end{tabular}

“_" means the corresponding value does not exist.

In the previous study[6], $\mathrm{W}-\mathrm{W} \mathrm{H}$-bonds were found to prevail in the methanol/water mixtures. Moreover, Table 3 shows that $f_{k}^{k}$ takes an overwhelming majority of $f_{k}^{n}$. It means that most water molecules are surrounded by water with the help of H-bonds. Due to the quantitative advantage of water molecules, there is no opportunity for most water molecules to be surrounded by methanol. This is beneficial to the formation of large water cluster. Water molecules are interested in H-bonding interaction, and less than $1.5 \%$ water molecules are isolated from other molecules as shown in Table 3 .

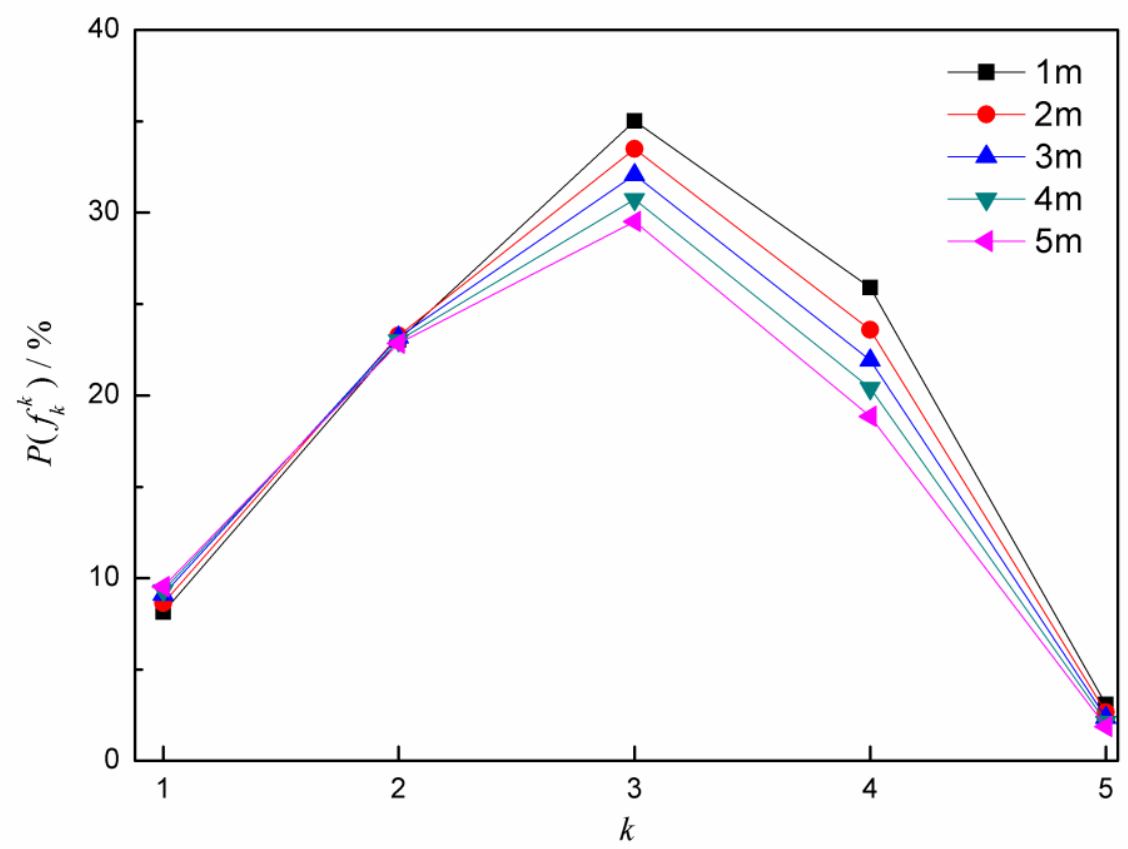

Fig. 1. Distributions of the water molecules in the H-bonding states $f_{k}^{k}(k=1 \sim 5)$ for 
the mixtures of different concentrations at $298 \mathrm{~K}$. The solid lines are drawn as a guide to the eye.

Fig. 1 depicts the distributions of the water molecules only $\mathrm{H}$-bonded to water in the binary mixtures with the concentration from 1 to $5 \mathrm{~m}$ at $298 \mathrm{~K}$. It is shown that methanol has greater effect on $f_{3}^{3}$ and $f_{4}^{4}$ than the other H-bonding states. The H-bonding states (i.e. $f_{1}^{1}, f_{2}^{2}$ and $f_{5}^{5}$ ) seem to have inconspicuous change with concentration. It should be noted that the water molecules in state $f_{5}^{5}$ is relatively few, thus the change of $f_{5}^{5}$ with methanol content may not noticeable. To demonstrate this, the maximum changes with concentration for the three $\mathrm{H}$-bonding states are calculated, which are $17 \%, 2 \%$ and $66 \%$ for $f_{1}^{1}, f_{2}^{2}$ and $f_{5}^{5}$, respectively. The dependence on the methanol concentration suggests that the added methanol molecules gradually disrupt the H-bonding network connected by water molecules, and the empty H-bonding sites are provided for the formation of new H-bonds. The empty H-bonding sites are mainly occupied by methanol molecules in the form of W-M H-bonds. Thus the number of W-M H-bonds increases with the concentration increases, which has been reported in Ref[1]. That is to say, the increasing concentration of methanol leads to simple H-bonding transfer from water-water complex to methanol-water complex[22]. Besides, the H-bonding states $f_{3}^{3}$ and $f_{4}^{4}$ are the typically local structures in the aqueous environment[23]. The formation of the two local structures by H-bonds makes it possible to produce a percolating network through the aqueous system[24], resulting in many amazing and unique properties[25]. The decreasing $f_{3}^{3}$ and $f_{4}^{4}$ implies the gradual disruption of the percolating H-bond network of water. 


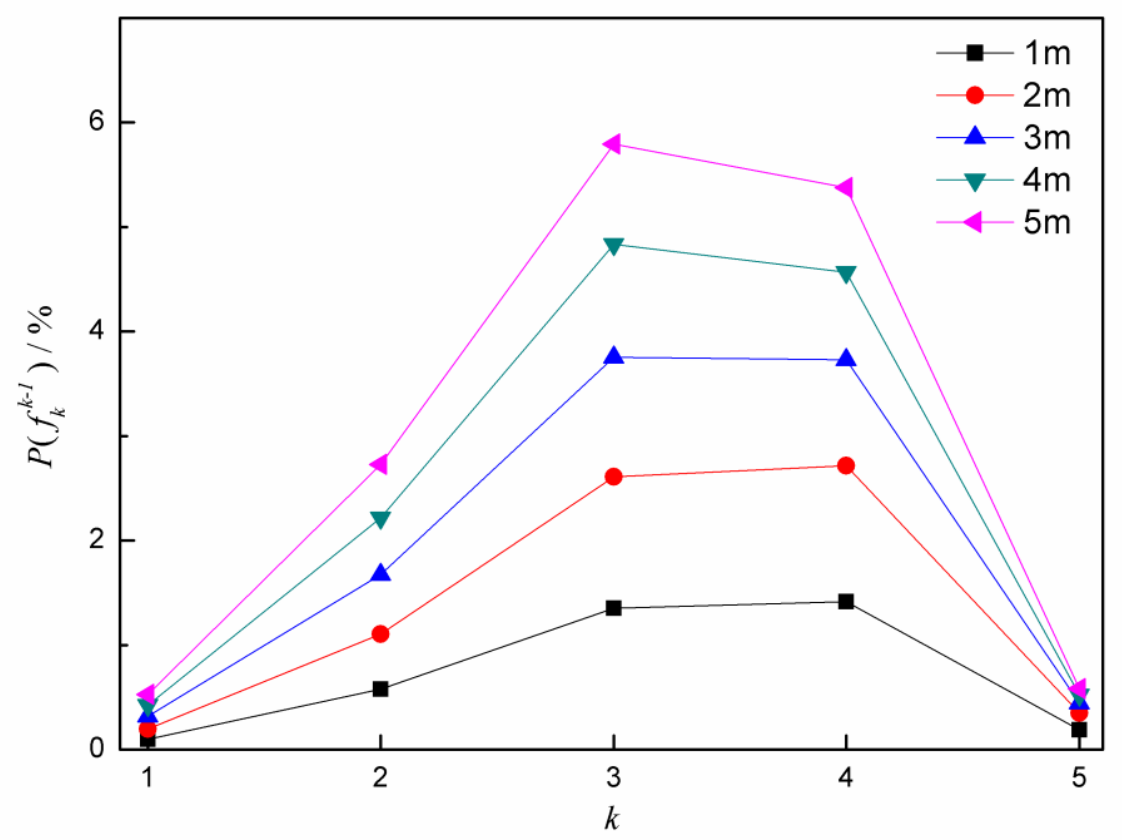

Fig. 2. Distributions of the water molecules in the H-bonding states $f_{k}^{k-1} \quad(k=1 \sim 5)$ for the mixtures of different concentrations at $288 \mathrm{~K}$.

The concentration also has influence on the H-bonding state $f_{k}^{k-1}$ in addition to $f_{k}^{k}$. Fig. 2 shows the percentage of the water molecules in the H-bonding states $f_{k}^{k-1}$ in the mixtures of different concentrations. It is shown that the number of the water in $f_{k}^{k-1}$ increases with the increasing concentration. At the value at $k=3$, $f_{3}^{2}$ has the largest increase from $1.4 \%$ to $5.8 \%$, which is less than the decrease by $f_{3}^{3}$; while $f_{3}^{1}$ only has an increase from $0.017 \%$ to $0.405 \%$ as the molality increases from 1 to $5 \mathrm{~m}$, which is ten times less than that of $f_{3}^{2}$; the increase for is three orders of magnitude less than $f_{3}^{2}$. This is similar with the case for the value $k=4$. The data not shown in Fig. 1 and 2 can be found in Table 3 and the supplemental information (Table S1). The increase in the percentage of $f_{k}^{n}(n<k)$ indicates that the methanol molecules gradually occupy the role of water in the H-bonding network. The 
inconsistence in the quantities of the decrease by $f_{k}^{k}$ and the overall increase by $f_{k}^{n}(n<k)$ shows that the loss of the W-W H-bonds is not compensated by the formation W-M H-bonds. This is consistent with the previous study[6], which made a statistical average of the H-bonds in the methanol/water mixtures. The following paragraphs will further study the effect of methanol on the H-bonding states of water.

3.1.4. Effects of temperature and concentration on $f_{0}^{0}$

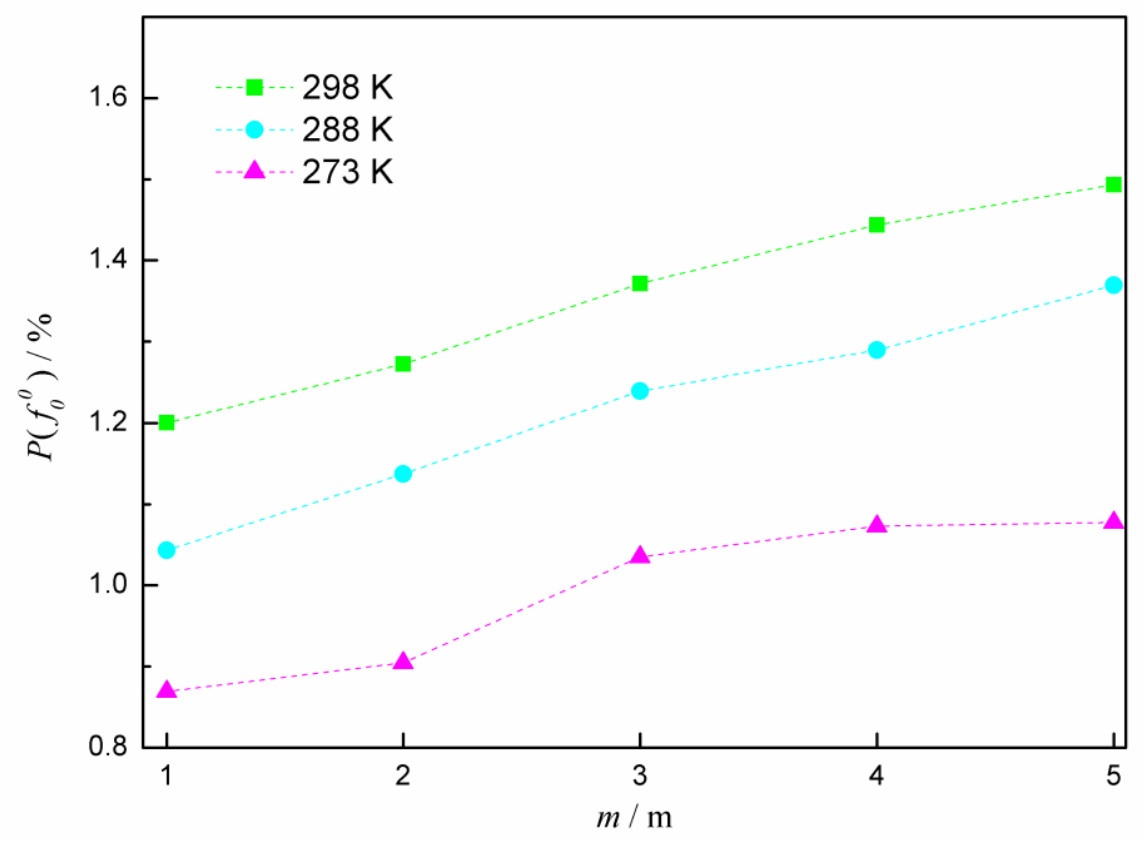

Fig. 3. Distributions of the water molecules in the H-bonding state $f_{0}^{0}$ under the conditions of different concentrations and temperatures. The dashed lines are drawn as a guide to the eye.

For the sake of brevity, the supplemental information (Table S1 S3) provides the results of $P\left(f_{k}^{n}\right)$ of the mixtures with the concentrations $1 \sim 5 \mathrm{~m}$ at different temperatures. It is worth noting that rising the temperature reduces the amount of the H-bonding complex of one water molecule with more than one methanol molecule. Moreover, the rising temperature also increases the probability of isolated water 
molecules (i.e. the water in the H-bonding state $f_{0}^{0}$ ). This is consistent with that in pure water as reported previously[26]. The dependences on temperature are mainly due to the thermal motion. Fig. 3 clearly depicts the results of $P\left(f_{0}^{0}\right)$ as a function of molality at three temperatures. Isolated water molecules even exist in pure systems without any other solutes at different temperatures[26]. It is noted that the concentration also affects the percentage of the water molecules in the H-bonding state $f_{0}^{0}$, which increases as the concentration increases. The phenomenon is similar to that in the glycerol aqueous solution[27]. In this study, concentration is another factor affecting the presence of isolated water molecules. It is expected that increasing amount of methanol molecules in the mixture would inhibit the H-bonds formed by water molecules to some extent.

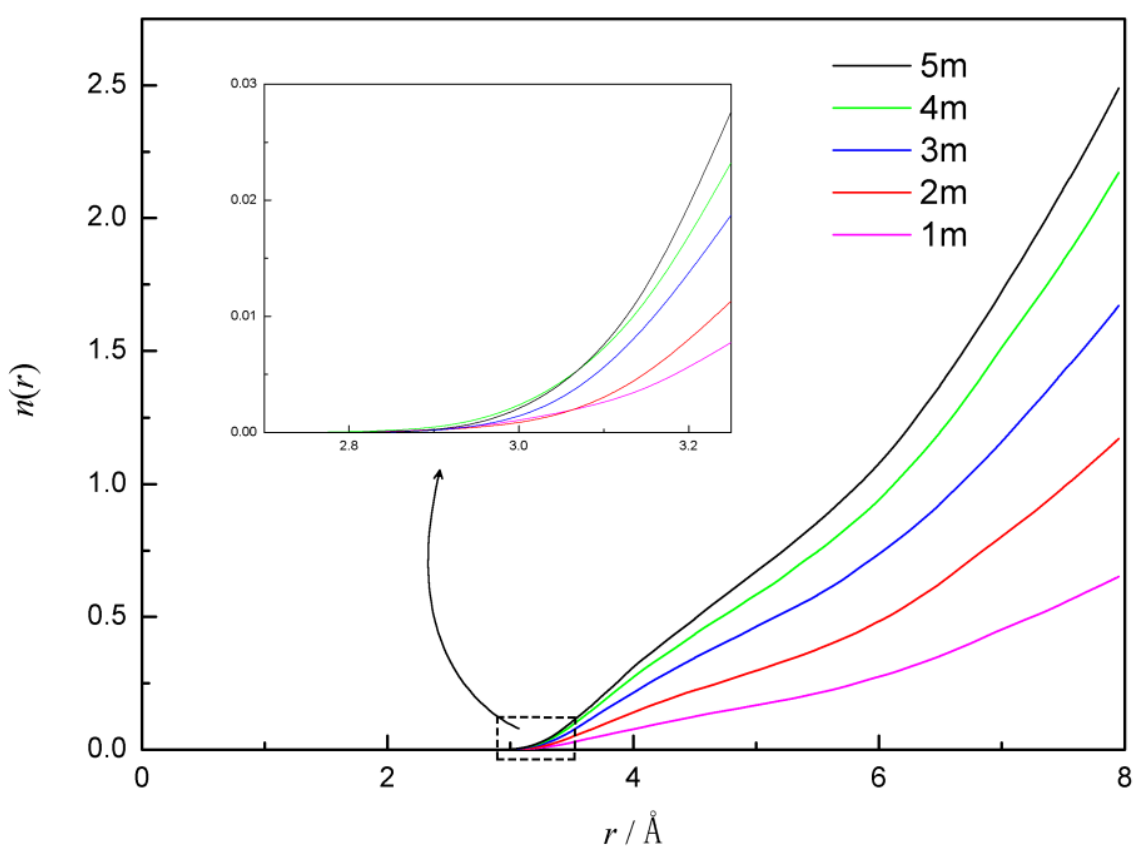

Fig. 4. The coordination number of methanol around the water molecules in the H-bonding state $f_{0}^{0}$ in the mixtures of different concentrations when the temperature equals to $298 \mathrm{~K}$. Partial enlargement of the result is shown in the inlet graph.

To explore the effect of concentration on the presence of isolated water molecules, Fig. 4 displays the distribution of the number of the methanol molecules 
around the water in the H-bonding state $f_{0}^{0}$ in the mixtures with the concentration from 1 to $5 \mathrm{~m}$. In the statistics, the positions of the methanol molecules are substituted with the carbon atoms. As expected, the probability of the presence of the methanol molecules around the water will increase with increasing the concentration. The size of methanol molecule is larger than that of water, and there is only one hydrophobic group in one methanol molecule. The two facts produce steric inhibition of the $\mathrm{W}-\mathrm{W}$ H-bonds. From Fig. 4, it can be seen that the coordination number of methanol around the water in the H-bonding state $f_{0}^{0}$ increases as the concentration increases. The presence of methanol around one water molecule reduces the probability of the formation of the $\mathrm{W}-\mathrm{W} \mathrm{H}-$ bonds due to the abovementioned steric inhibition.

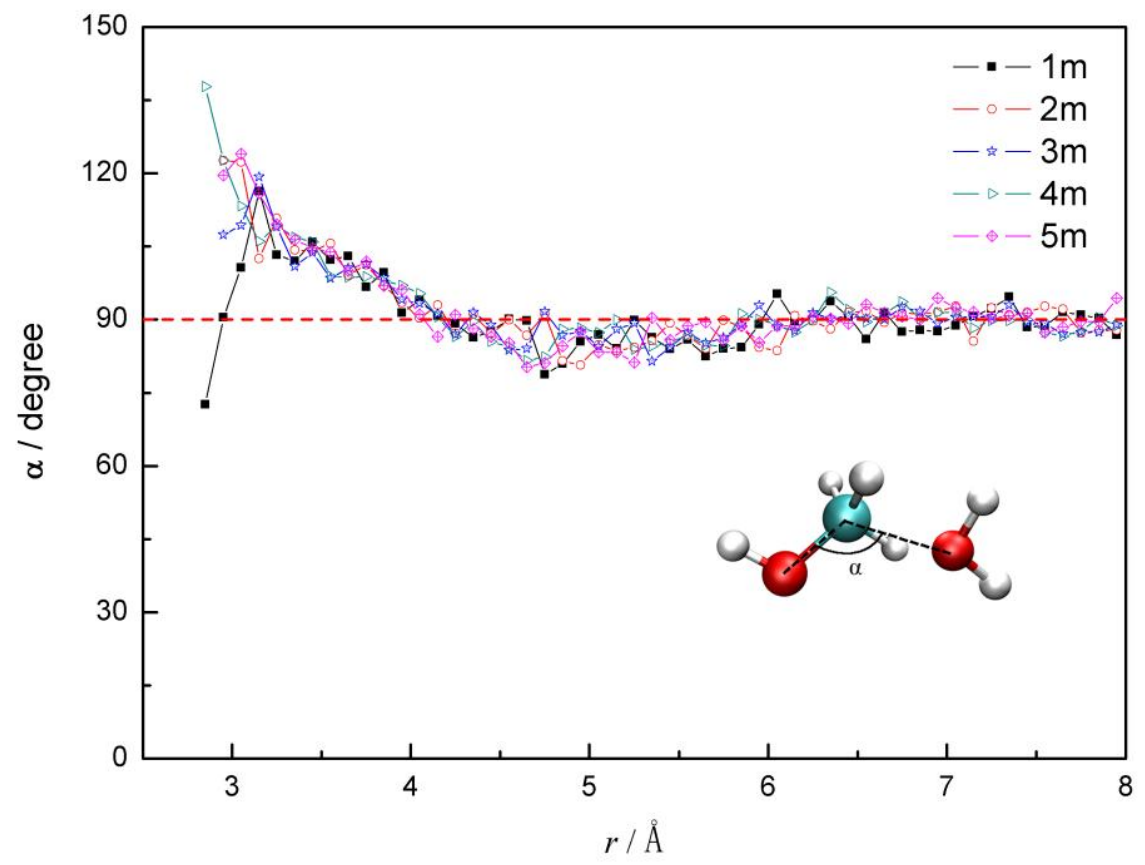

Fig. 5. The average value of the orientational angle of the methanol around the water in the H-bonding state $f_{0}^{0}$ as a function of the distance between the methanol and water molecules. The snapshot shows the orientational angle $\alpha$ formed by methanol and water, where oxygen atoms are colored as red, hydrogen as white, and carbon as cyan.

The formation of the W-W H-bonds is impeded by the adjacent methanol 
molecules. However, at what distance from the central water molecule do the adjacent methanol take effect on the H-bonding state of the water molecule. In order to explore the effective range of the steric effect resulted from methanol, Fig. 5 further describes the orientation of the methanol molecules in the vicinity of the water molecules in $f_{0}^{0}$. The character $\alpha$ denotes the angle between the covalent bond C-O of methanol and the vector from the carbon atom of methanol to the oxygen atom of water, where the carbon atom is the vertex of the angle. There is no distribution of methanol molecules around the central water molecules within the distance about $2.9 \AA$, beyond which methanol appears in the vicinity of the central water molecule. This is consistent with the results in Fig. 4. The orientational angle reaches almost the maximum value larger than $90^{\circ}$ when methanol appears near the water in $f_{0}^{0}$. As the distance from the central water increases, $\alpha$ gradually decreases to the value $90^{\circ}$. At the distance about $4 \AA$, $\alpha$ begins to fluctuate around the value $90^{\circ}$, implying that the methanol molecules have random orientations around the water in $f_{0}^{0}$. Thus, the methanol molecules have little effect on the central water beyond the distance of $4 \AA$. Within the distance of $4 \AA$, the orientational angle tends to be greater than $90^{\circ}$, indicating that the hydrophobic group of the methanol molecule tends to face to the central water molecule. The intruded hydrophobic group $\left(-\mathrm{CH}_{3}\right)$ into the hydration shell of the water molecule in $f_{0}^{0}$ blocks the formation of $\mathrm{H}$-bonds with other molecules, which is the main reason of the quantitative inconsistence in the $\mathrm{H}$-bonding transfer from $\mathrm{W}-\mathrm{W}$ to $\mathrm{W}-\mathrm{M} \mathrm{H}$-bonds as the concentration increases.

In this section, we calculated the constituents of the H-bonds for each water molecule, and made a detailed statistic analysis of the water molecules in different H-bonding states. Table 3 shows that each water molecule could possess two types of H-bonds in different proportions, among which the H-bonding states $f_{k}^{k} \quad(\mathrm{k}=1 \sim 5)$ take the dominant role. It is worth noting that in addition to the temperature effect, the concentration of methanol has effect on the presence of the water molecule in the H-bonding state $f_{0}^{0}$. According to the above discussion based on Fig. 4 and 5, we 
can get the information that methanol is beneficial to the presence of the isolated water molecules by means of its hydrophobic group. Thus as the concentration of methanol increases, the number of isolated water molecules (i.e. the water molecules in the H-bonding state $f_{0}^{0}$ ) increases.

\subsection{Self-diffusivity of water in different H-bonding states}

It has been reported that the self-diffusion behavior of water molecules has a close correlation with the H-bonding interaction in the methanol/water mixtures[6]. In this section, we will further investigate the effects of different H-bonding types on the self-diffusion motion of the water molecules in different H-bonding states. According to the definition of H-bonding state, the self-diffusion of the water molecules could be separately studied. In the last section, the results of $f_{k}^{n}$ shows that some H-bonding states account for very low percentages. Thus this section focuses on the H-bonding states with high percentages, and further clarifies the self-diffusivity of the water molecules in different H-bonding states.

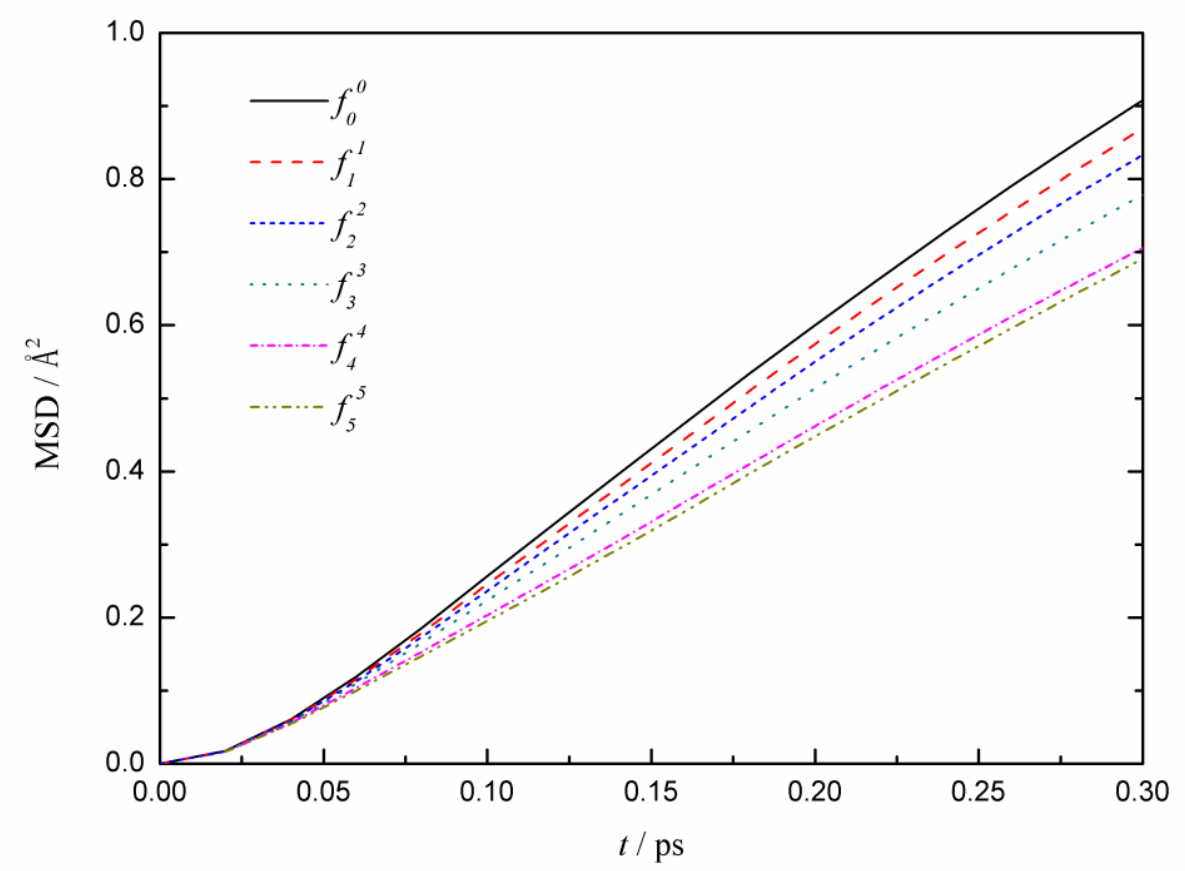

Fig. 6. MSD as a function of simulation time for the water molecules at different 
initial hydrogen H-bonding states of $f_{k}^{k}(k=0 \sim 5)$ in the mixture of with the molality of $3 \mathrm{~m}$ at $298 \mathrm{~K}$.

It is difficult to determine the self-diffusion coefficients of the water molecules in different H-bonding states due to two reasons reported previously[6]. Thus Einstein-Smoluchowski relation does not work in the analysis of the water molecules classified by the definition of H-bonding state. Meanwhile, in the previous work[6], the H-bonding state of water is an mean effect of different types of H-bonds. To further understand the mean effect, Fig. 6 presents the self-diffusion motions of the water molecules only with W-W H-bonds and without any other types of H-bonds (i.e. in the H-bonding states $f_{k}^{k}$ ) in the mixture of $3 \mathrm{~m}$. We only trace the water molecules initially in the interested H-bonding states for a short time of $0.3 \mathrm{ps}$. The time range is chosen according to the H-bonding lifetimes of the $\mathrm{W}-\mathrm{W}$ and $\mathrm{W}-\mathrm{M}$ H-bonds[6]. The W-W H-bond was found to have the shortest H-bonding lifetime of $0.318 \mathrm{ps}$ in the methanol/water mixture with the molality of $1 \mathrm{~m}$ at $298 \mathrm{~K}[6]$. The MSD results in the figure shows that the water molecules with more $\mathrm{H}$-bonds diffuse more slowly than those with less H-bonds. This further proves that H-bonding interaction slows down the self-diffusivity of the H-bonded water molecules[6, 10]. 


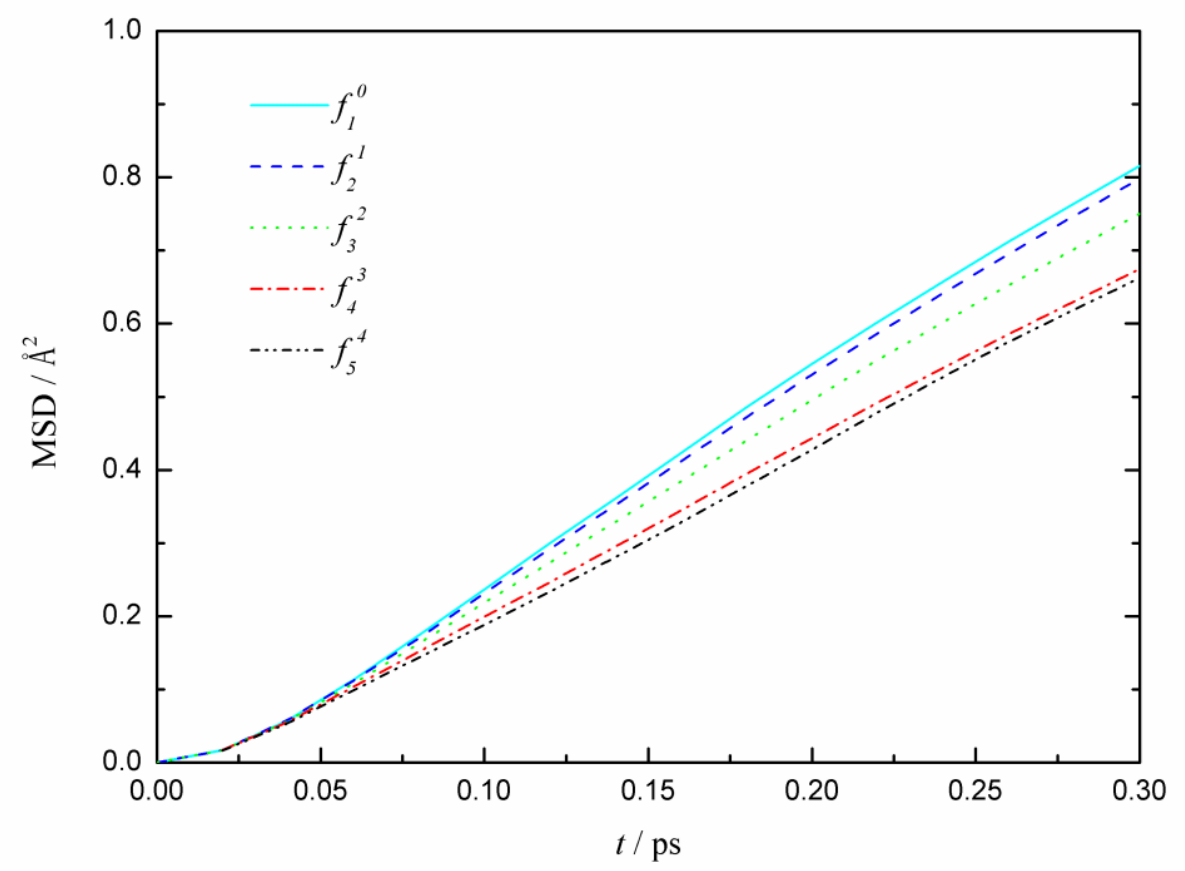

Fig. 7. MSD as a function of simulation time for the water molecules in different initial H-bonding states of $f_{k}^{k-1} \quad(k=1 \sim 5)$ in the mixture of the molality of $3 \mathrm{~m}$ at $298 \mathrm{~K}$.

Fig. 7 depicts the MSDs of the water molecules in the H-bonding states of $f_{k}^{k-1}(k=1 \sim 5)$ as a function of time from 0 to $0.3 \mathrm{ps}$. The results shown in the figure also reveal that although the water molecules are H-bonded with one methanol molecule, their self-diffusion motion is slowed down as the number of W-W H-bonds increases. The difference between $f_{k}^{k}$ and $f_{k}^{k-1}$ can be considered as a substitution of one water molecule with one methanol molecule. Note that the MSD value of $f_{k}^{k}$ is larger than the corresponding value of $f_{k}^{k-1}$. It is shown in Fig. 8 that as the value $n$ in $f_{k}^{n}$ decreases, the self-diffusivity of the water molecules decreases. Fig. 8 only depicts part of $f_{3}^{n}$ and $f_{4}^{n}$ due to the negligible percentage of the other H-bonding states of $f_{3}^{n}$ and $f_{4}^{n}$, as shown in Table 3. It is seen obviously that the MSD values of the water in $f_{3}^{n}$ holds the increasing order of $f_{3}^{1}$ 
$<f_{3}^{2}<f_{3}^{3}$. It implies that methanol helps to slow down the self-diffusion of the H-bonded water molecules. The water in $f_{4}^{n}$ follows the same order in quantity of $f_{4}^{2}<f_{4}^{3}<f_{4}^{4}$. The MSDs of $f_{3}^{n}$ are all larger than those of $f_{4}^{n}$, indicating that water with more H-bonds diffuses more slowly.

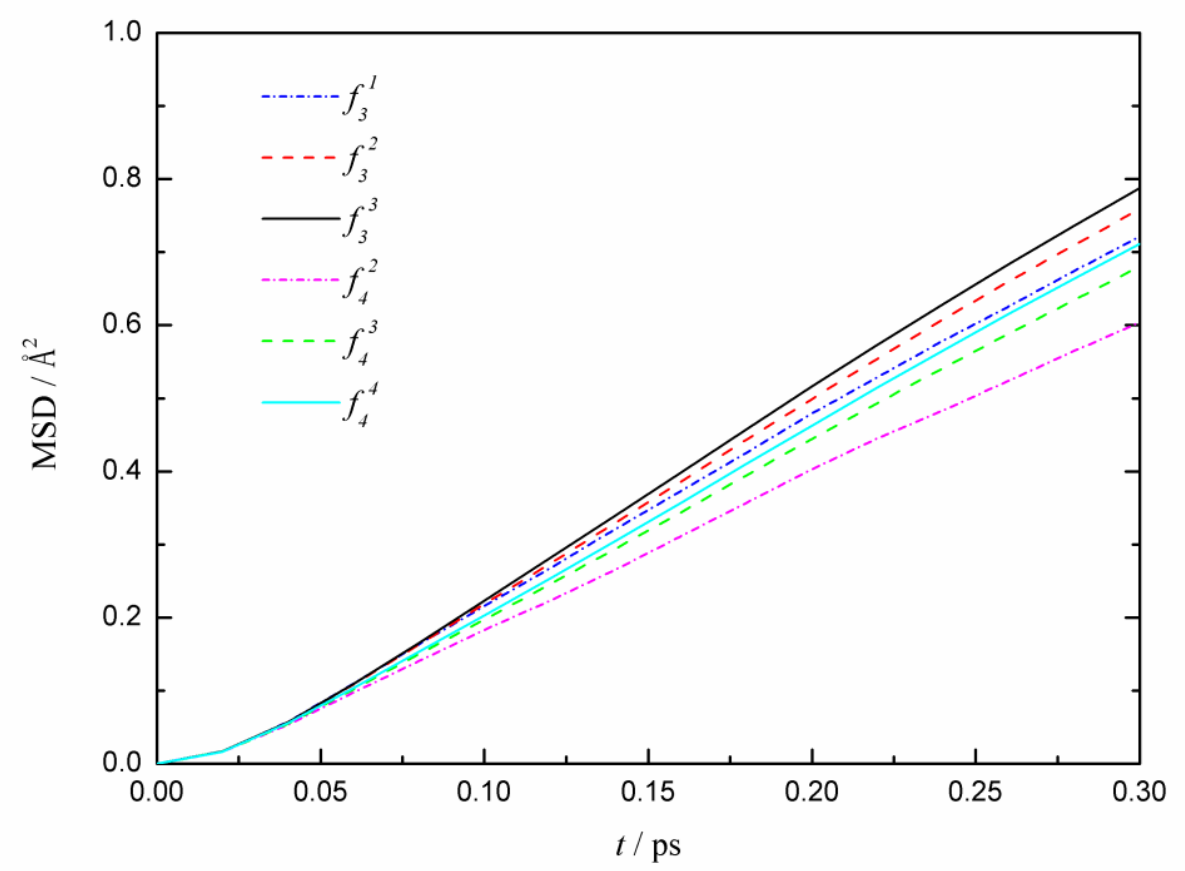

Fig. 8. MSDs of the water molecules in the H-bonding states $f_{3}^{n}$ and $f_{4}^{n}$ in the mixture of $1 \mathrm{~m}$ at $298 \mathrm{~K}$.

To explore the relationship between concentration and water self-diffusivity, Fig. 9 depicts the MSD values of the water molecules in $f_{k}^{k}$ at the moment of $t=0.3 \mathrm{ps}$ for the mixtures with the concentration $m=1 \sim 5 \mathrm{~m}$ at the temperature $288 \mathrm{~K}$. As the concentration increases, the water molecules show a decreasing trend in the MSD value. In some cases fluctuation appears, but the decreasing tendency could be noticed. Other H-bonding states of $f_{k}^{n}$ follow the same dependence on concentration as that of $f_{k}^{k}$, thus it is not shown for brevity. The concentration-dependence of the self-diffusion coefficient is also resulted from the block of the solute size effect[10] and the clusters of hydrophobic group[28]. 


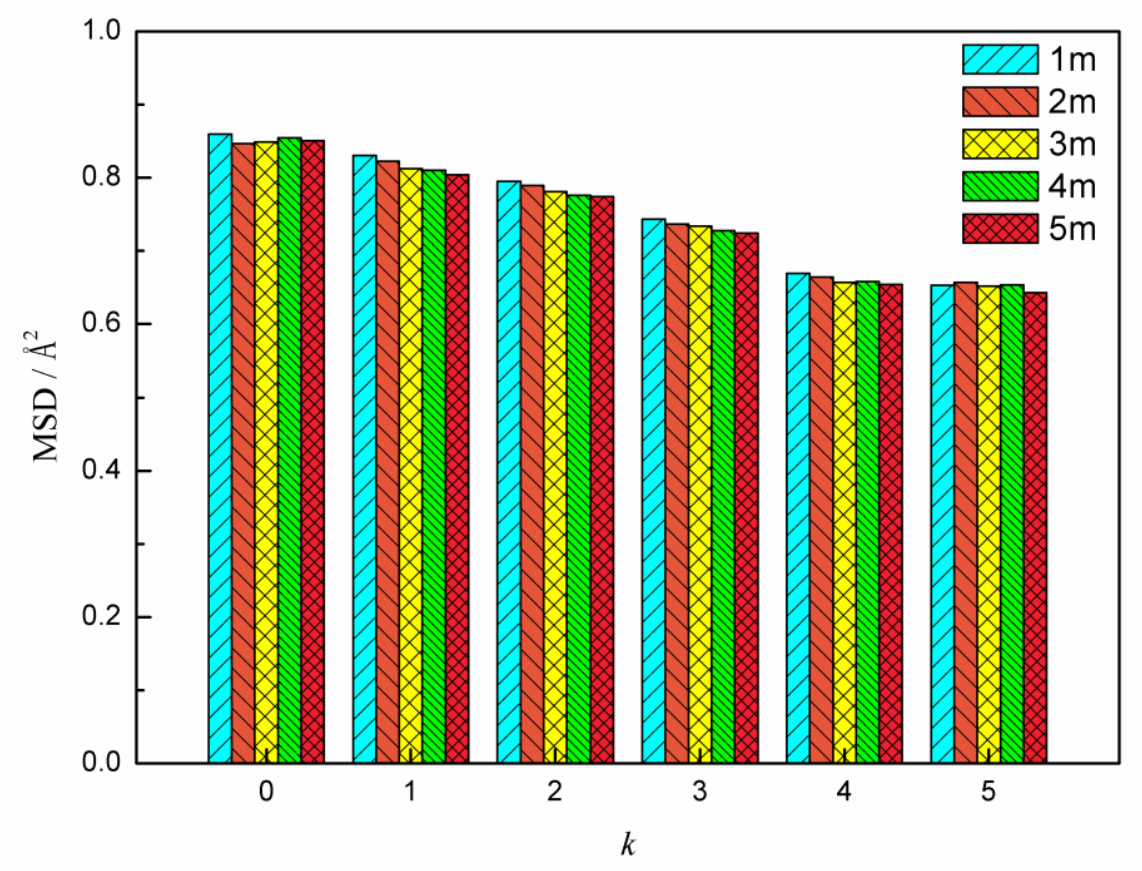

Fig. 9. MSDs of the water molecules in the H-bonding states $f_{k}^{k}$ at $t=0.3$ ps for the mixture with the molality ranging from 1 to $5 \mathrm{~m}$ at $288 \mathrm{~K}$.

Temperature also has effect on the water self-diffusion. Fig. 10 provides the MSD values of the water molecules of $f_{k}^{k}$ at the time $t=0.3$ ps for the binary mixture of $5 \mathrm{~m}$ at three temperatures. For all the H-bonding states of $f_{k}^{k}$, the MSD values increase with the temperature increases. This is mainly due to the thermal motion, which facilitates the self-diffusion motion and the escape from H-bonding attraction[21]. Thus raising temperature leads to the disruption of the H-bonding network formed by water molecules[29]. Next section will interpret the dependence of water self-diffusivity on H-bonding and temperature in the point view of pair energy. 


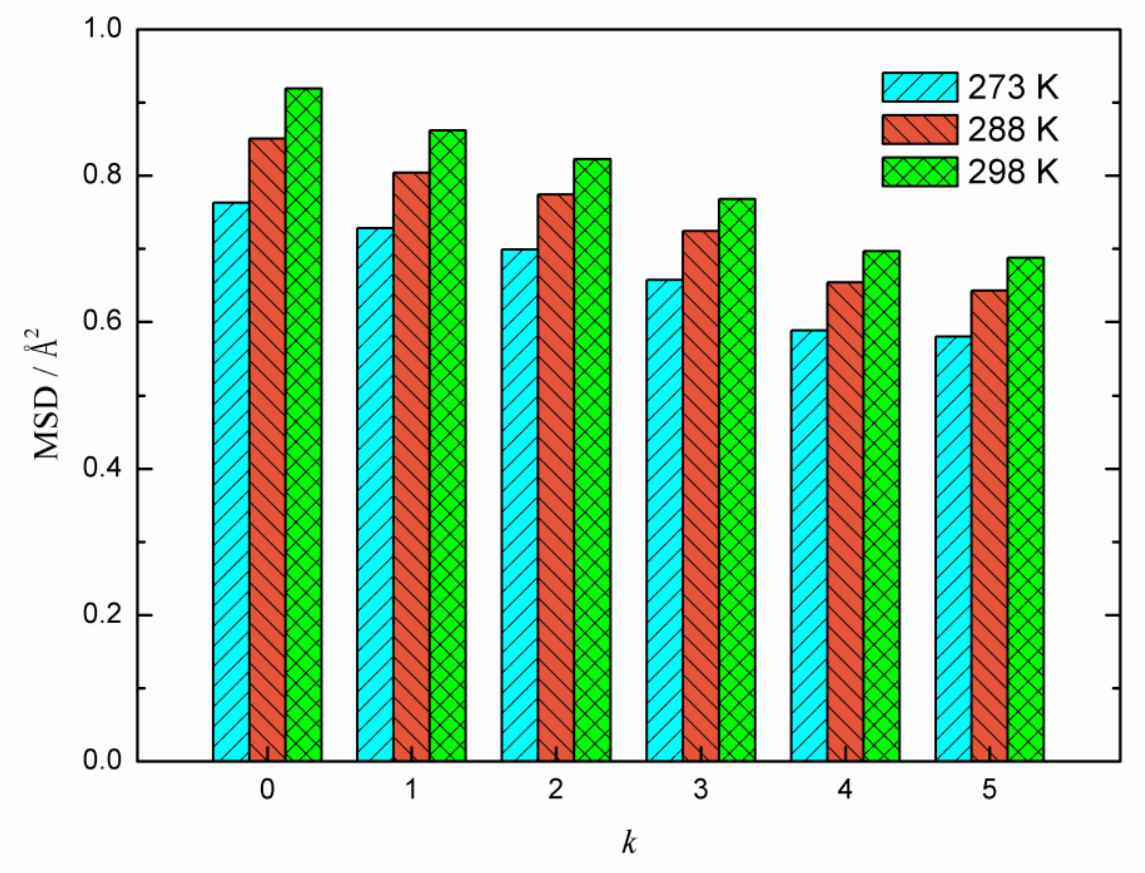

Fig. 10. MSDs of the water molecules in the H-bonding states $f_{k}^{k}$ at $t=0.3$ ps for the mixture with the molality of $5 \mathrm{~m}$ at 273,288 and $298 \mathrm{~K}$.

\subsection{Pair energy of H-bonded molecules}

The strength of the intermolecular interactions in liquid system can be characterized by the pair energy between two molecules[23, 30]. Herein, the pair energies between the H-bonded molecules are calculated to make a deeper analysis of the dependence of intermolecular association on the H-bond type (W-W and W-M H-bonds). For each binary mixture, we have considered in the analysis by computing the mean pair energies of the H-bonded W-W and W-M molecular pairs, which are listed in Table 4.

In Table 4, the mean pair energy of the H-bonded W-M pairs shows larger absolute value than that of the $\mathrm{W}-\mathrm{W}$ pairs in each methanol/water mixture. This implies that the H-bonding interaction between water and methanol molecules is more stable than that between water molecules, resulting in longer lifetime of W-M H-bond than W-W H-bond[6]. As a result, the self-diffusion of water molecules has a 
decreasing trend as the methanol concentration increases due to the stronger attraction of the W-M H-bond. Thus increasing the concentration helps increase the probability of the formation of W-M H-bond, which enhances the bound effect on water molecules and slows down the water self-diffusivity. The dependence of water self-diffusivity on concentration only takes effect within a limited concentration range. When the concentration increases to some extent, the self-diffusion motion of water will show an opposite trend[31]. This is resulted from the disruption of the percolating water H-bonding network $[22,32,33]$, the mechanism of which is beyond the scope of interest in this work. Although the greatest difference between $\varepsilon_{\mathrm{WM}}$ than $\varepsilon_{\mathrm{WM}}$ is 1.52 $\mathrm{kJ} / \mathrm{mol}$ in the mixture of $4 \mathrm{~m}$ at $298 \mathrm{~K}$, the total effect of methanol on the inhibition of water self-diffusion is still significant due to the large amount of $\mathrm{H}$-bonded pairs for the macroscopic case.

Table 4. Mean pair energies ( $\varepsilon_{\mathrm{Ww}}$ and $\varepsilon_{\mathrm{WM}}$ ) of the H-bonded molecules for W-W and W-M pairs in the mixtures with the concentration from 1 to $5 \mathrm{~m}$ at three temperatures.

The unit of the pair energy in the table is $\mathrm{kJ} / \mathrm{mol}$.

\begin{tabular}{|c|c|c|c|c|c|c|}
\hline$T(\mathrm{~K})-M(\mathrm{~m})$ & & 1 & 2 & 3 & 4 & 5 \\
\hline \multirow{2}{*}{273} & $\varepsilon_{\mathrm{WW}}$ & -17.49 & -17.51 & -17.49 & -17.58 & -17.92 \\
\hline & $\varepsilon_{\mathrm{WM}}$ & -18.35 & -18.74 & -18.50 & -17.78 & -18.37 \\
\hline \multirow{2}{*}{288} & $\varepsilon_{\mathrm{WW}}$ & -17.25 & -17.40 & -17.08 & -17.54 & -17.38 \\
\hline & $\varepsilon_{\mathrm{WM}}$ & -17.90 & -18.36 & -17.79 & -17.89 & -18.22 \\
\hline \multirow{2}{*}{298} & $\varepsilon_{\mathrm{WW}}$ & -17.12 & -17.23 & -17.17 & -17.23 & -17.24 \\
\hline & $\varepsilon_{\mathrm{WM}}$ & -17.98 & -18.72 & -18.14 & -18.75 & -18.32 \\
\hline
\end{tabular}

The mean pair energies $\varepsilon_{\mathrm{WW}}$ and $\varepsilon_{\mathrm{WM}}$ seem to have little dependence on the concentration. The data in Table 4 has small fluctuation as the concentration increases. Thus the addition of methanol would not affect the strength of the H-bonding interaction between different molecules. However, as the temperature increases, both $\varepsilon_{\mathrm{WW}}$ and $\varepsilon_{\mathrm{WM}}$ show decreasing tendency. This is due to the fact that the temperature-enhanced thermal motion facilitates the escape of the molecules from the H-bonding interaction. Thus, raising the temperature is conductive to promoting the self-diffusion of water molecules. This is consistent with the information shown in Fig. 10. 


\subsection{Cluster lifetime}

Water and methanol clusters simultaneously exist in their binary mixtures. As the concentration increases, the aggregation of methanol molecules is enhanced and the water H-bonding network is gradually disrupted[6]. The previous study provides us the dependence of cluster size on concentration, while the dynamic behavior of cluster is also an interesting property. Banerjee and Bagchi[34] adopted an effective strategy in the analysis of the clusters of amphiphilic solute in water, motivating us to study the cluster lifetime.

In this section, we will investigate the dynamic nature of water and methanol clusters, respectively. Definition of cluster should be determined before the analysis of cluster lifetime. For water cluster, if any two water molecules are H-bonded according to the H-bonding criterion (Section 3.3.1), they are considered to belong to the same water cluster. As for methanol cluster, the connectivity of methanol molecules is defined by the RDF between $\mathrm{CH}_{3}$ groups of any two methanol molecules in the mixtures. The definition of the cluster of the amphiphilic molecules is similar with the previous work $[6,32,34]$. In the methanol/water mixtures, the position of the first minimum of the RDF was measured at $5.8 \AA[6]$, within which two methanol molecules are considered to be connected by a dummy chemical bond. To characterize the dynamic behavior of the water and methanol clusters, the time correlation function is defined for the connected molecules $i$ and $j$ as

$$
C(t)=\frac{\left\langle h_{i j}(t) h_{i j}(0)\right\rangle}{\left\langle h_{i j}(0) h_{i j}(0)\right\rangle}
$$

where, $h_{i j}(t)$ is an instantaneous number of connected pairs with respect to time $t$. if two initially connected molecules $i$ and $j$ continuously connect to each other for a period of time $t$, then $h_{i j}(t)=1$ is defined for the two molecules; otherwise, the variable $h_{i j}(t)$ is set to 0 , and the two molecules will not be taken into account. The connection between molecules $i$ and $j$ denotes H-bonding interaction for water cluster or hydrophobic aggregation for methanol cluster. 

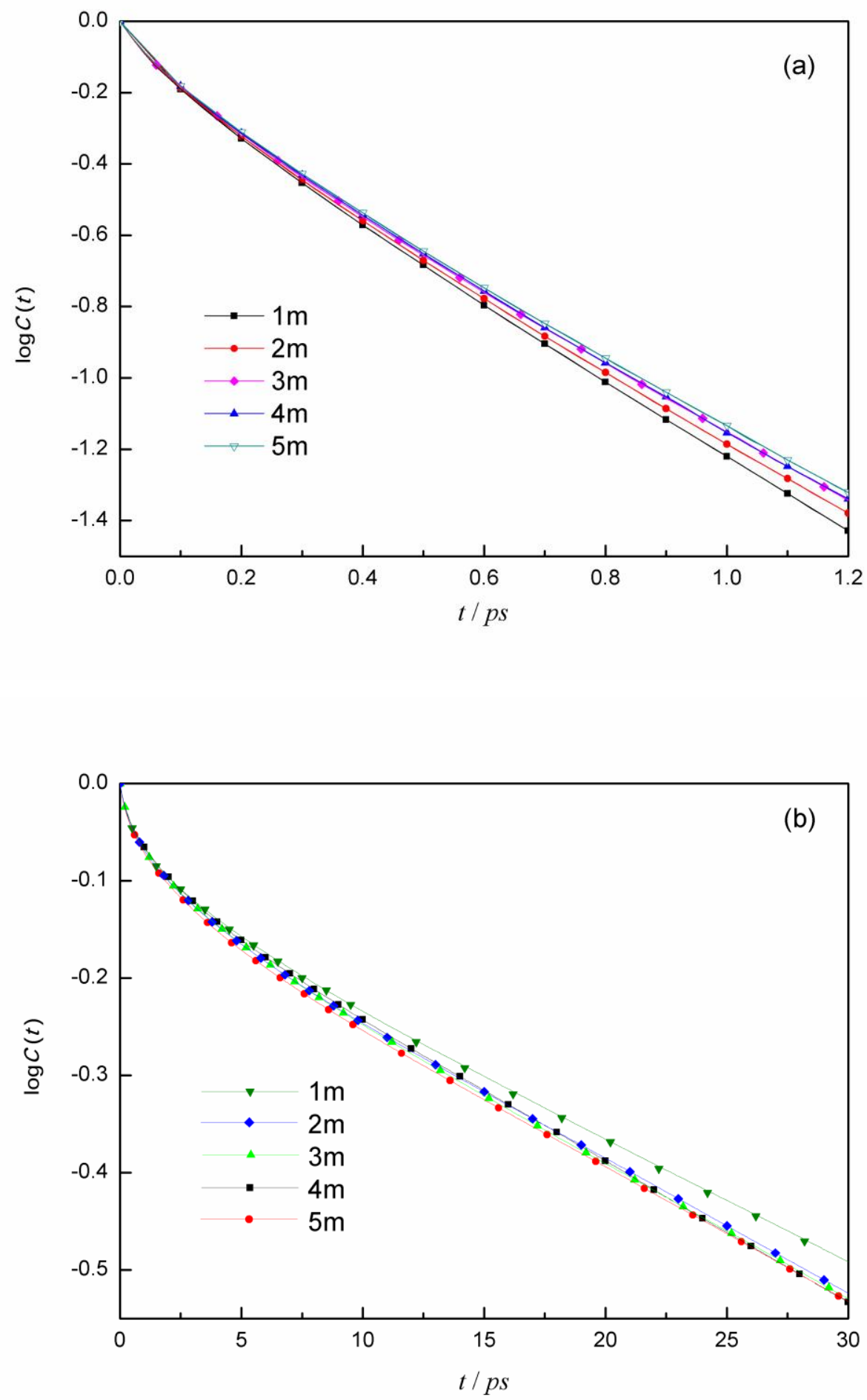

Fig. 11. Correlation function $C(t)$ [eq. (1)] for water (a) and methanol (b) clusters at different concentrations. Several selected data are shown for better representations.

The time correlation function describes the stability of the connections in the water and methanol clusters. Fig. 11(a) and 11(b) depict the plot of logarithm of the 
correlation functions $C(t)$ of the water and methanol clusters for the mixtures of different concentrations. The water cluster shows less stable than the methanol clusters. The correlation functions were fitted to the bi-exponential equation, Eq. (2). The fitting parameters for the water and methanol clusters in their binary mixtures of different concentrations are tabulated in Table 5 and 6 , respectively.

$$
f(t)=a \exp \left(-\frac{t}{\tau_{1}}\right)+(1-a) \exp \left(-\frac{t}{\tau_{2}}\right)
$$

For water cluster, it is found that melting lifetime is about one magnitude lower than breaking lifetime for each methanol/water mixture. The melting and breaking lifetimes have been explained by Banerjee and Bagchi[34]. It is shown in Table 5 that $a>0.5$ means water prefers to single particle evaporation. For methanol cluster, melting lifetime is also about one magnitude lower than breaking lifetime, and both lifetimes are one magnitude higher than the lifetimes of water cluster, respectively. However, it is known from Table 6 that methanol resists any single particle evaporation. This is similar to the result of the tert-butyl alcohol aqueous solution[34]. The resistance to formation of monomers implies that one water molecule $\mathrm{H}$-bonded to methanol is likely to move with methanol cluster, enhancing the resistance to its thermal motion.

Table.5 The fitting parameters of Eq. (2) for the correlation function $C(t)$ of the water clusters in the methanol/water mixtures of different concentrations.

\begin{tabular}{cccc}
\hline$m(\mathrm{~m})$ & $a$ & $\tau_{1}(\mathrm{ps})$ & $\tau_{2}(\mathrm{ps})$ \\
\hline 1 & 0.756 & 0.070 & 0.389 \\
2 & 0.748 & 0.074 & 0.403 \\
3 & 0.738 & 0.079 & 0.419 \\
4 & 0.752 & 0.074 & 0.416 \\
5 & 0.750 & 0.074 & 0.423 \\
\hline
\end{tabular}

Table.6 The fitting parameters of Eq. (2) for the correlation function $C(t)$ of the 
methanol clusters in the methanol/water mixtures of different concentrations.

\begin{tabular}{cccc}
\hline$m(\mathrm{~m})$ & $a$ & $\tau_{1}(\mathrm{ps})$ & $\tau_{2}(\mathrm{ps})$ \\
\hline 1 & 0.20 & 1.34 & 32.46 \\
2 & 0.19 & 1.26 & 29.20 \\
3 & 0.21 & 1.32 & 30.42 \\
4 & 0.20 & 1.32 & 29.81 \\
5 & 0.22 & 1.37 & 30.23 \\
\hline
\end{tabular}

\section{Conclusion}

In order to further investigate the H-bonding effect on water self-diffusion, several MD simulations have been performed for the methanol/water mixtures under the conditions of different concentrations and temperatures. Firstly, we defined the $\mathrm{H}$-bonding state for water according to its different numbers of $\mathrm{W}-\mathrm{W}$ and $\mathrm{W}-\mathrm{M}$ H-bonds. On the basis of the definition, we have calculated the distributions of the water molecules in all the defined H-bonding states for different concentrations and temperatures. It was found that the H-bonding states $f_{k}^{k}$ and $f_{k}^{k-1}$ take the dominant role. Especially, $f_{3}^{3}$ has advantage in quantity over the other H-bonding states of $f_{k}^{k}$. The amounts of water molecules in $f_{4}^{4}$ are still evident for all the mixtures. It implies that water molecules show local tetrahedral structure in the studied concentration range. Then the paper emphasizes on the interpretation of the dependence of isolated water molecules on concentration by computing the orientational angle of the methanol molecules around the central water in $f_{0}^{0}$. Due to the insertion of the methanol's hydrophobic group $-\mathrm{CH}_{3}$ into the hydration shell, the central water is apt to be away from other W-W H-bonding partners. Thus the number of isolated water molecules increases as the concentration increases.

Secondly, the MSDs as a function of time were calculated for the H-bonding states with numerical superiority, such as $f_{k}^{k}$ and $f_{k}^{k-1}$. The water molecules with 
more H-bonds diffuses more slowly, consistent with our previous study[6]. Fig. 8 further exhibits the dependence of self-diffusion on different types of H-bonds. W-M H-bond seems to have more retarding effect on water self-diffusion than W-W H-bond. To interpret this, the third section made an analysis in the viewpoint of pair energy between the H-bonded molecules. For all the mixtures at different temperatures, the values of the pair energy $\varepsilon_{\mathrm{WM}}$ are always larger than that of $\varepsilon_{\mathrm{WW}}$, indicating that W-M H-bond shows more attraction to water than W-W H-bond. It also indicates the fact that the lifetime of $\mathrm{W}-\mathrm{M} \mathrm{H}$-bond is longer than $\mathrm{W}-\mathrm{W} \mathrm{H}$-bond. As temperature increases, the increasing strength of water facilitates an escape from the restriction of H-bonds. Thus water molecules diffuse more slowly in concentrated mixtures of methanol due to the fact that the energy $\varepsilon_{\mathrm{WM}}$ is larger than $\varepsilon_{\mathrm{Ww}}$. Moreover, cluster analysis shows that methanol cluster is more stable than water cluster. Then we can imagine that one water inside the methanol cluster diffuses out with more difficulty than in the easily "melting" water cluster. Based on the previous study[6], the present paper further explores the H-bonding effect on water self-diffusion in order to help understand the mechanism of the dependences of the water self-diffusion motion on temperature and concentration in the methanol aqueous solution.

\section{Supplementary Data}

Percentages $\left(P\left(f_{k}^{n}\right) \%\right)$ of the water molecules at different $\mathrm{H}$-bonding states $\left(f_{k}^{n}\right)$ in the methanol/water mixture with different concentrations at the temperatures of 298, 288 and $273 \mathrm{~K}$ are shown in Table S1 S3.

\section{Acknowledge}

This research has been supported by The National Science Fund for Distinguished Young Scholars of China(No. 21125628), The Fundamental Research Funds for the Central Universities of China(No. DUT14RC(3)077) and The State Key Program of National Natural Science of China(No. 21236006). We thank to 


\section{Computing Center in Department of Energy and Power Engineering of Dalian}

University of Technology for providing parallel computing environment.

\section{References}

[1] N. Zhang, W. Li, C. Chen, J. Zuo, L. Weng, Molecular Dynamics Investigation of the Effects of Concentration on Hydrogen Bonding in Aqueous Solutions of Methanol, Ethylene Glycol and Glycerol, B. Kor. Chem. Soc. 34 (2013), 9.

[2] R. Devanathan, N. Idupulapati, M. Dupuis, Molecular modeling of the morphology and transport properties of two direct methanol fuel cell membranes: Phenylated sulfonated poly(ether ether ketone ketone) versus Nafion, J. Mater. Res. 27 (2012), 1927-1938.

[3] Y. Zeng, C. Wang, X. Zhang, S. Ju, Solvation structure and dynamics of Li+ ion in liquid water, methanol and ethanol: A comparison study, Chem. Phys. 433 (2014), 89-97.

[4] Y.-P. Zeng, X.-M. Zhu, Z.-H. Yang, Car-Parrinello Molecular Dynamics Simulations of Microstructure Properties of Liquid Water, Methanol and Ethanol, Acta Phys-chim. Sin. 27 (2011), 2779-2785.

[5] S.K. Pattanayak, S. Chowdhuri, Effects of methanol on the hydrogen bonding structure and dynamics in aqueous N-methylacetamide solution, J. Mol. Liq. 194 (2014), 141-148.

[6] N. Zhang, W. Li, C. Chen, J. Zuo, L. Weng, Molecular dynamics study on water self-diffusion in aqueous mixtures of methanol, ethylene glycol and glycerol: investigations from the point of view of hydrogen bonding, Mol. Phys. 111 (2013), 939-949.

[7] T. Weitkamp, J. Neuefeind, H.E. Fischer, M.D. Zeidler, Hydrogen bonding in liquid methanol at ambient conditions and at high pressure, Mol. Phys. 98 (2000), 125-134.

[8] Y. Zhong, G.L. Warren, S. Patel, Thermodynamic and structural properties of methanol-water solutions using nonadditive interaction models, J. Comput. Chem. 29 (2008), 1142-1152.

[9] L. Dougan, S.P. Bates, R. Hargreaves, J.P. Fox, J. Crain, J.L. Finney, V. Reat, A.K. Soper, Methanol-water solutions: A bi-percolating liquid mixture, J. Chem. Phys. 121 (2004), 6456-6462.

[10] J.T. Su, P.B. Duncan, A. Momaya, A. Jutila, D. Needham, The effect of hydrogen bonding on the diffusion of water in n-alkanes and n-alcohols measured with a novel single microdroplet method, $J$. Chem. Phys. 132 (2010), 044506-044508.

[11] R.G.M. van der Sman, Predictions of Glass Transition Temperature for Hydrogen Bonding Biomaterials, J. Phys. Chem. B 117 (2013), 16303-16313.

[12] D.-X. Li, B.-L. Liu, Y.-s. Liu, C.-1. Chen, Predict the glass transition temperature of glycerol-water binary cryoprotectant by molecular dynamic simulation, Cryobiology 56 (2008), 114-119.

[13] S. Roy, S. Banerjee, N. Biyani, B. Jana, B. Bagchi, Theoretical and Computational Analysis of Static and Dynamic Anomalies in Water-DMSO Binary Mixture at Low DMSO Concentrations, $J$. Phys. Chem. B 115 (2010), 685-692.

[14] C. Chen, W.Z. Li, Y.C. Song, L.D. Weng, N. Zhang, Concentration dependence of water self-diffusion coefficients in dilute glycerol-water binary and glycerol-water-sodium chloride ternary solutions and the insights from hydrogen bonds, Mol. Phys. 110 (2011), 283-291.

[15] J. Wang, T. Hou, Application of molecular dynamics simulations in molecular property prediction II: Diffusion coefficient, J. Comput. Chem. 32 (2011), 3505-3519.

[16] D.J. Keffer, P. Adhangale, The composition dependence of self and transport diffusivities from 
molecular dynamics simulations, Chem. Eng. J. 100 (2004), 51-69.

[17] D.J. Keffer, C.Y. Gao, B.J. Edwards, On the Relationship between Fickian Diffusivities at the Continuum and Molecular Levels, J. Phys. Chem. B 109 (2005), 5279-5288.

[18] W.L. Jorgensen, J. Chandrasekhar, J.D. Madura, R.W. Impey, M.L. Klein, Comparison of simple potential functions for simulating liquid water, J. Chem. Phys. 79 (1983), 926-935.

[19] A.D. MacKerell, D. Bashford, Bellott, R.L. Dunbrack, J.D. Evanseck, M.J. Field, S. Fischer, J. Gao, H. Guo, S. Ha, D. Joseph-McCarthy, L. Kuchnir, K. Kuczera, F.T.K. Lau, C. Mattos, S. Michnick, T. Ngo, D.T. Nguyen, B. Prodhom, W.E. Reiher, B. Roux, M. Schlenkrich, J.C. Smith, R. Stote, J. Straub, M. Watanabe, J. Wiórkiewicz-Kuczera, D. Yin, M. Karplus, All-Atom Empirical Potential for Molecular Modeling and Dynamics Studies of Proteins†, J. Phys. Chem. B 102 (1998), 3586-3616.

[20] N. Zhang, W. Li, C. Chen, J. Zuo, L. Weng, Evaluation of the Application of Hydrogen Bonding Criteria to DMSO Aqueous Solution, Acta Phys. Chim. Sin. 29 (2013), 1891-1899.

[21] L. Weng, C. Chen, J. Zuo, W. Li, Molecular Dynamics Study of Effects of Temperature and Concentration on Hydrogen-Bond Abilities of Ethylene Glycol and Glycerol: Implications for Cryopreservation, J. Phys. Chem. A 115 (2011), 4729-4737.

[22] N. Zhang, W. Li, C. Chen, J. Zuo, Molecular dynamics simulation of aggregation in dimethyl sulfoxide-water binary mixture, Comput. Theor. Chem. 1017 (2013), 126-135.

[23] I. Bako, T. Megyes, S. Balint, V. Chihaia, M.-C. Bellissent-Funel, H. Krienke, A. Kopf, S.-H. Suh, Hydrogen bonded network properties in liquid formamide, J. Chem. Phys. 132 (2010), 014506.

[24] H.-J. Kim, J.M. Kim, Cyclic topology in complex networks, Phys. Rev. E 72 (2005), 036109.

[25] F.H. Stillinger, Theory and Molecular Models for Water, in: Advances in Chemical Physics, John Wiley \& Sons, Inc., 2007, 1-101.

[26] J. Marti, J.A. Padro, E. Guardia, Molecular dynamics simulation of liquid water along the coexistence curve: Hydrogen bonds and vibrational spectra, J. Chem. Phys. 105 (1996), 639-649.

[27] C. Chen, W.Z. Li, Y.C. Song, J. Yang, Hydrogen bonding analysis of glycerol aqueous solutions: A molecular dynamics simulation study, J. Mol. Liq. 146 (2009), 23-28.

[28] S. Banerjee, S. Roy, B. Bagchi, Enhanced Pair Hydrophobicity in the Water-Dimethylsulfoxide (DMSO) Binary Mixture at Low DMSO Concentrations, J. Phys. Chem. B 114 (2010), 12875-12882.

[29] D.C. Rapaport, Hydrogen bonds in water -- Network organization and lifetimes, Mol. Phys. 50 (1983), 1151-1162.

[30] D. Swiatla-Wojcik, Evaluation of the criteria of hydrogen bonding in highly associated liquids, Chem. Phys. 342 (2007), 260-266.

[31] Z.J. Derlacki, A.J. Easteal, A.V.J. Edge, L.A. Woolf, Z. Roksandic, Diffusion coefficients of methanol and water and the mutual diffusion coefficient in methanol-water solutions at 278 and $298 \mathrm{~K}$, J. Phys. Chem. 89 (1985), 5318-5322.

[32] S. Banerjee, R. Ghosh, B. Bagchi, Structural Transformations, Composition Anomalies and a Dramatic Collapse of Linear Polymer Chains in Dilute Ethanol-Water Mixtures, J. Phys. Chem. B 116 (2012), 3713-3722.

[33] J.J. Towey, A.K. Soper, L. Dougan, Molecular Insight Into the Hydrogen Bonding and Micro-Segregation of a Cryoprotectant Molecule, J. Phys. Chem. B 116 (2012), 13898-13904.

[34] S. Banerjee, B. Bagchi, Stability of fluctuating and transient aggregates of amphiphilic solutes in aqueous binary mixtures: Studies of dimethylsulfoxide, ethanol, and tert-butyl alcohol, J. Chem. Phys. 139 (2013), - 